\title{
Some Inequalities for the Maximum Modulus of Rational Functions
}

\author{
Robert Gardner, ${ }^{1}$ Narendra Kumar Govil $\mathbb{D}^{1},{ }^{2}$ and Prasanna Kumar ${ }^{3}$ \\ ${ }^{1}$ Department of Mathematics and Statistics, East Tennessee State University, Johnson City, TN 37614, USA \\ ${ }^{2}$ Department of Mathematics and Statistics, Auburn University, Auburn, AL 36849, USA \\ ${ }^{3}$ Department of Mathematics, Birla Institute of Technology and Science Pilani, K K Birla Goa Campus, Sancoale,
} Goa 403726, India

Correspondence should be addressed to Narendra Kumar Govil; govilnk@auburn.edu

Received 6 June 2021; Accepted 18 August 2021; Published 3 September 2021

Academic Editor: Raúl E. Curto

Copyright (c) 2021 Robert Gardner et al. This is an open access article distributed under the Creative Commons Attribution License, which permits unrestricted use, distribution, and reproduction in any medium, provided the original work is properly cited.

\begin{abstract}
For a polynomial $p(z)$ of degree $n$, it follows from the maximum modulus theorem that $\max _{|z|=R \geq 1}|p(z)| \leq R^{n} \max _{|z|=1}|p(z)|$. It was shown by Ankeny and Rivlin that if $p(z) \neq 0$ for $|z|<1$, then $\max _{|z|=R \geq 1}|p(z)| \leq\left(\left(R^{n}+1\right) / 2\right) \max _{|z|=1}|p(z)|$. In 1998, Govil and Mohapatra extended the above two inequalities to rational functions, and in this paper, we study the refinements of these results of Govil and Mohapatra.
\end{abstract}

\section{Introduction and Statement of Results}

Let $\mathrm{P}_{n}$ denote the set of all complex algebraic polynomials $p$ of degree at most $n$ and let $p^{\prime}$ be the derivative of $p$. For a function $f$ defined on the unit circle $\mathbb{T}=\{z|| z \mid=1\}$ in the complex plane $\mathbb{C}$, set $\|f\|=\sup _{z \in \mathbb{T}}|f(z)|$, the Chebyshev norm of $f$ on $\mathbb{T}$.

Let $\mathbb{D}_{-}$denote the region strictly inside $\mathbb{T}$ and $\mathbb{D}_{+}$be the region strictly outside $\mathbb{T}$. For $a_{v} \in \mathbb{C}, v=1,2, \ldots, n$, let

$$
\begin{aligned}
& w(z)=\prod_{v=1}^{n}\left(z-a_{v}\right), \\
& B(z)=\prod_{v=1}^{n} \frac{\left(1-\bar{a}_{v} z\right)}{\left(z-a_{v}\right)},
\end{aligned}
$$

being the Blaschke product, and

$$
\mathscr{R}_{n}=\mathscr{R}_{n}\left(a_{1}, a_{2}, \ldots, a_{n}\right)=\left\{\frac{p(z)}{w(z)} \mid p \in \mathrm{P}_{n}\right\} .
$$

Then, $\mathscr{R}_{n}$ is the set of rational functions with possible poles at $a_{1}, a_{2}, \ldots, a_{n}$ and having a finite limit at $\infty$. Also, note that $B(z) \in \mathscr{R}_{n}$.

\subsection{Definitions}

(i) For polynomial $p(z)=\sum_{v=0}^{n} \alpha_{v} z^{v}$, the conjugate transpose (reciprocal) $p^{*}$ of $p$ is defined by

$$
\begin{aligned}
p^{*}(z) & =z^{n} \overline{p\left(\frac{1}{\bar{z}}\right)}=z^{n} \bar{p}\left(\frac{1}{z}\right) \\
& =\bar{\alpha}_{0} z^{n}+\bar{\alpha}_{1} z^{n-1}+\cdots+\bar{\alpha}_{n} .
\end{aligned}
$$

(ii) For rational function $r(z)=p(z) / w(z) \in \mathscr{R}_{n}$, the conjugate transpose, $r^{*}$, of $r$ is defined by

$$
r^{*}(z)=B(z) \overline{r\left(\frac{1}{\bar{z}}\right)}=B(z) \bar{r}(1 / z) .
$$


(iii) The polynomial $p \in \mathrm{P}_{n}$ is self-inversive if $p^{*}(z)=$ $\lambda p(z)$ for some $\lambda \in \mathbb{T}$.

(iv) The rational function $r \in \mathscr{R}_{n}$ is self-inversive if $r^{*}(z)=\lambda r(z)$ for some $\lambda \in \mathbb{T}$.

It is easy to verify that if $r \in \mathscr{R}_{n}$ and $r=p / w$, then $r^{*}=p^{*} / w$, and hence, $r^{*} \in \mathscr{R}_{n}$. So, $p / w$ is self-inversive if and only if $p$ is self-inversive.

For some related results on cubic rational splines, see Abbas et al. [1, 2].

If $p \in \mathrm{P}_{n}$, then it is well known that

$$
\max _{|z|=R \geq 1}|p(z)| \leq R^{n}\|p\| \text {. }
$$

This inequality is an immediate consequence of the maximum modulus theorem. Furthermore, if $p \in \mathrm{P}_{n}$ has all its zeros in $\mathbb{T} \cup \mathbb{D}_{+}$, then

$$
\max _{|z|=R \geq 1}|p(z)| \leq \frac{R^{n}+1}{2}\|p\| .
$$

Inequality (6) is due to Ankeny and Rivlin [3]. Both inequalities (5) and (6) are sharp; inequality (5) becomes equality for $p(z)=\lambda z^{n}$, where $\lambda \in \mathbb{C}$, and inequality (6) becomes equality for $p(z)=\alpha z^{n}+\beta$, where $|\alpha|=|\beta|$.

Govil and Mohapatra [4] gave a result analogous to inequality (5), but for rational functions, it is as follows.

Theorem 1. If

$$
r(z)=\frac{p(z)}{w(z)}=\frac{p(z)}{\prod_{v=1}^{n}\left(z-a_{v}\right)} \in \mathscr{R}_{n},
$$

is a rational function with $\left|a_{v}\right|>1$ for $1 \leq v \leq n$, then for $|z| \geq 1$,

$$
|r(z)| \leq\|r\||B(z)| \text {. }
$$

This result is best possible and equality holds for $r(z)=\lambda \prod_{v=1}^{n}\left(1-\bar{a}_{v} z\right) /\left(z-a_{v}\right)=\lambda B(z)$, where $\lambda \in \mathbb{C}$.

In the same paper, Govil and Mohapatra [4] also proved a result given as follows, that is analogous to inequality (6) for rational functions.

Theorem 2. Let

$$
r(z)=\frac{p(z)}{w(z)}=\frac{p(z)}{\prod_{v=1}^{n}\left(z-a_{v}\right)} \in \mathscr{R}_{n},
$$

with $\left|a_{v}\right|>1$ for $1 \leq v \leq n$. If all the zeros of $r$ lie in $\mathbb{U} \cup \mathbb{\mathbb { D } _ { + }}$, then for $|z| \geq 1$,

$$
|r(z)| \leq\|r\| \frac{|B(z)|+1}{2} .
$$

This result is best possible and equality holds for the rational function $r(z)=\alpha B(z)+\beta$, where $|\alpha|=|\beta|$.

In this paper, we firstly present the following refinement of the above Theorem 1. Here, $p(z)=\sum_{v=0}^{n} \alpha_{v} z^{v}$ is a polynomial of degree at most $n$.

Theorem 3. If

$$
r(z)=\frac{p(z)}{w(z)}=\frac{p(z)}{\prod_{v=1}^{n}\left(z-a_{v}\right)} \in \mathscr{R}_{n},
$$

is a rational function with $\left|a_{v}\right|>1,1 \leq v \leq n$, then for $|z| \geq 1$,

$$
|r(z)| \leq\|r\||B(z)|\left\{1-\frac{\left(\|r\|-\left|r^{*}(0)\right|\right)(|z|-1)}{\left|r^{*}(0)\right|+|z|\|r\|}\right\} .
$$

The result is best possible and equality holds for $r(z)=\lambda B(z)$, where $\lambda \in \mathbb{C}$.

Remark 1. It is clear that Theorem 3 sharpens Theorem 1 . Also, we can use Theorem 3 to derive a sharpening form of Bernstein's inequality for polynomials. For this, let $p(z)=$ $\sum_{v=0}^{n} \alpha_{v} z^{v}$ be a polynomial of degree $n$. Then, $r(z)=p(z) / \prod_{v=1}^{n}\left(z-a_{v}\right) \in \mathscr{R}_{n}$, and hence by Theorem 3, for $|z| \geq 1$,

$$
\left|\frac{r(z)}{B(z)}\right|=\left|\frac{p(z)}{\prod_{v=1}^{n}\left(1-\bar{a}_{v} z\right)}\right| \leq\|r\|\left\{1-\frac{\left(\|r\|-\left|r^{*}(0)\right|\right)(|z|-1)}{\left|r^{*}(0)\right|+|z|\|r\|}\right\} .
$$

If $z^{*}$ on $|z|=1$ is such that

$$
\|r\|=\left|r\left(z^{*}\right)\right|=\frac{\left|p\left(z^{*}\right)\right|}{\left|\prod_{v=1}^{n}\left(z^{*}-a_{v}\right)\right|}
$$

then we get from (13)

Since $\quad p(z)=\sum_{v=0}^{n} \alpha_{v} z^{v} \quad$ and $\quad r^{*}(z)=p^{*}(z) /$ $\prod_{v=1}^{n}\left(z-a_{v}\right)$, we get $\left|r^{*}(0)\right|=\left|\alpha_{n}\right| / \prod_{v=1}^{n}\left|a_{v}\right|$, and therefore, from (16), we have for $|z|>1$, 


$$
\begin{aligned}
& \qquad|p(z)| \leq\left|p\left(z^{*}\right)\right| \prod_{v=1}^{n} \mid \frac{1-\bar{a}_{v} z}{z^{*}-a_{v}}\left\{1-\frac{\left(\left|p\left(z^{*}\right)\right|-\left|\alpha_{n}\right| \prod_{v=1}^{n}\left|\left(z^{*}-a_{v}\right) / a_{v}\right|\right)(|z|-1)}{\left|\alpha_{n}\right| \prod_{v=1}^{n}\left|\left(z^{*}-a_{v}\right) / a_{v}\right|+|z \|| p\left(z^{*}\right) \mid}\right\} . \\
& \qquad\left|B\left(\frac{1}{z}\right)\right|=\prod_{j=1}^{n}\left|\frac{z-\bar{a}_{j}}{1-z a_{j}}\right| \leq 1, \quad \text { for }|z| \geq 1 . \\
& \text { for all }\left|a_{v}\right| \geq 1 \text {, where } 1 \leq v \leq n \text {, making } \quad \\
& \leq v \leq n \text {, we get that, for }|z| \geq 1,
\end{aligned}
$$

Since (17) holds for all $\left|a_{v}\right| \geq 1$, where $1 \leq v \leq n$, making $\left|a_{v}\right| \longrightarrow \infty$, where $1 \leq v \leq n$, we get that, for $|z| \geq 1$,

$$
|p(z)| \leq\left|p\left(z^{*}\right) \| z\right|^{n}\left\{1-\frac{\left(\left|p\left(z^{*}\right)\right|-\left|\alpha_{n}\right|\right)(|z|-1)}{\left|\alpha_{n}\right|+\left|z \| p\left(z^{*}\right)\right|}\right\} .
$$

We show in Lemma 2, in Section 2, that the expression on the right hand side of (18) is an increasing function of $\left|p\left(z^{*}\right)\right|$. Note that $\left|p\left(z^{*}\right)\right| \neq 0$, for if $\left|p\left(z^{*}\right)\right|=0$, then $\left|r\left(z^{*}\right)\right| \neq\|r\|$. On applying this fact to (18), we get that, for $|z| \geq 1$,

$$
|p(z)| \leq\|p\||z|^{n}\left\{1-\frac{\left(\|p\|-\left|\alpha_{n}\right|\right)(|z|-1)}{\left|\alpha_{n}\right|+|z|\|p\|}\right\},
$$

which is equivalent to that for $|z|=R \geq 1$, we have

$$
|p(z)| \leq R^{n}\left\{1-\frac{\left(\|p\|-\left|\alpha_{n}\right|\right)(R-1)}{\left|\alpha_{n}\right|+R\|p\|}\right\}\|p\| .
$$

This rate of growth result for a polynomial, which is a sharpening of Bernstein inequality, first appeared as Lemma 3 of [5].

Before we proceed to the proof of Theorem 3, we state the following result recently proved by Mir [6] and which is a refinement of Theorem 2 .

Theorem 4. Let $\quad r(z)=p(z) / w(z)=p(z) / \prod_{v=1}^{n}(z-$ $\left.a_{v}\right) \in \mathscr{R}_{n}$, with $\left|a_{v}\right|>1$ for $1 \leq v \leq n$. If all the zeros of $r$ lie in $\mathbb{T} \cup \mathbb{D}_{+}$, then for $|z| \geq 1$,

$$
|r(z)| \leq\|r\|\left\{\frac{|B(z)|+1}{2}\right\}-\left\{\frac{|B(z)|-1}{2}\right\} \min _{|z|=1}|r(z)| .
$$

We omit the proof of this theorem since it is already proved in the paper due to Mir [6]. However, related to this, we make the following two remarks.

Remark 2. It is clear that, in case $\min _{|z|=1}|r(z)|=0$, the above Theorem 4 reduces to Theorem 2. Also, it has been claimed by Mir [6] that, in all other cases except when $\min _{|z|=1}|r(z)|=0$, it gives a bound that is sharper than the one obtainable from Theorem 2. Although this claim seems to be correct but to justify this, it is necessary to show that $|B(z)| \geq 1$ for $|z| \geq 1$, which we show as follows.

Since $\left|a_{v}\right|>1$ for $1 \leq v \leq n$, the Blaschke product

$$
B(z)=\prod_{j=1}^{n}\left(\frac{1-\bar{a}_{j} z}{z-a_{j}}\right)
$$

is analytic in $|z| \leq 1$. Furthermore, on $|z|=1$, we have $|B(z)|=1$; hence, by the maximum modulus principle, we have $|B(z)| \leq 1$ for $|z| \leq 1$, which clearly implies
But, the above is equivalent to

$$
\prod_{j=1}^{n}\left|\frac{1-a_{j} z}{z-\bar{a}_{j}}\right| \geq 1, \quad \text { for }|z| \geq 1,
$$

which implies

$$
|B(\bar{z})|=\prod_{j=1}^{n}\left|\frac{1-\bar{a}_{j} \bar{z}}{\bar{z}-a_{j}}\right| \geq 1, \quad \text { for }|z| \geq 1 .
$$

The above clearly gives that $|B(z)| \geq 1$ for $|\bar{z}| \geq 1$, from which the desired inequality follows since the two sets $\{z:|z| \geq 1\}$ and $\{z:|\bar{z}| \geq 1\}$ are the same.

Remark 3. If in Theorem 4, we multiply both sides of (21) by $\prod_{v=1}^{n} a_{v}$ and then make each $a_{v}$ go to infinity, we get the following result due to Aziz and Dawood [7].

Theorem 5. Let $p(z)=\sum_{v=0}^{n} \alpha_{v} z^{v}$ be a polynomial of degree at most $n$. If $p(z)$ has no zeros in $|z|<1$, then for $R \geq 1$,

$$
\max _{|z|=R \geq 1}|p(z)| \leq\left\{\frac{R^{n}+1}{2}\right\} \max _{|z|=1}|p(z)|-\left\{\frac{R^{n}-1}{2}\right\} \min _{|z|=1}|p(z)| .
$$

The result is best possible and equality holds for $p(z)=\alpha z^{n}+\beta$, where $|\alpha|=|\beta|$.

The above Theorem 5 clearly sharpens inequality (6) in all cases except when $\min _{|z|=1}|p(z)|=0$, in which case it clearly reduces to (6).

Remark 4. It has come to our notice that, around the same time, our paper was submitted for publication, Milovanović and Mir [8] also submitted a paper containing Theorem 3. However, our proof of Theorem 3 is different than the one given in [8] because of our proof using the generalized form of Schwarz's lemma given in Nehari ([9], p. 167) (also, see Govil et al. ([10], p. 326)) while the proof in [8] uses a lemma due to Osserman [11].

Now, we proceed with the proof of Theorem 3, and in this regard, we present the following lemmas.

\section{Lemmas}

The following is a well-known generalization of Schwarz's lemma, given in Nehari ([9], p. 167) (also, see Govil et al. ([10], p. 326)).

Lemma 1. If $f$ is analytic inside and on the circle $|z|=1$, then for $|z| \leq 1$, 


$$
|f(z)| \leq\|f\| \frac{\|f\||z|+|f(0)|}{|f(0)\|z \mid+\| f \|} .
$$

Lemma 2. For $|z| \geq 1$, and $\alpha_{n} \in \mathbb{C}$, the function $g(x)=x\left\{1-\left(x-\left|\alpha_{n}\right|\right)(|z|-1) /\left(\left|\alpha_{n}\right|+|z| x\right)\right\}$ is an increasing function for $x>0$.

Proof. Note that

$$
\begin{aligned}
g(x) & =x\left\{1-\frac{\left(x-\left|\alpha_{n}\right|\right)(|z|-1)}{\left|\alpha_{n}\right|+|z| x}\right\} \\
& =x\left\{\frac{\left|\alpha_{n}\right||z|+x}{\left|\alpha_{n}\right|+|z| x}\right\},
\end{aligned}
$$

from which it clearly follows that for $x>0$, we have

$$
g^{\prime}(x)=\frac{|z| x^{2}+2\left|\alpha_{n}\right| x+\left|z \| \alpha_{n}\right|^{2}}{\left(\left|\alpha_{n}\right|+|z| x\right)^{2}} .
$$

Since the above expression is positive for $x>0$, the function $g(x)$ is increasing for $x>0$, as claimed.

\section{Proof of Theorem 3}

Since

$$
r(z)=\frac{p(z)}{w(z)}=\frac{p(z)}{\prod_{v=1}^{n}\left(z-a_{v}\right)} \in \mathscr{R}_{n},
$$

with $\left|a_{v}\right|>1$ for $1 \leq v \leq n$, the function

$$
r^{*}(z)=\frac{p^{*}(z)}{\prod_{v=1}^{n}\left(z-a_{v}\right)}
$$

is analytic in $|z| \leq 1$. Therefore, by Lemma 1 we get that, for $|z| \leq 1$,

$$
\left|r^{*}(z)\right| \leq\left\|r^{*}\right\| \frac{\left\|r^{*}\right\||z|+\left|r^{*}(0)\right|}{\left|r^{*}(0)\|z \mid+\| r^{*} \|\right.},
$$

and since $\left\|r^{*}\right\|=\|r\|$, inequality (32) is in fact equivalent to the inequality that, for $|z| \leq 1$,

$$
\left|r^{*}(z)\right| \leq\|r\| \frac{\|r\|\|z\|+\left|r^{*}(0)\right|}{\left|r^{*}(0)\|z \mid+\| r \|\right.} .
$$

Since by definition $r^{*}(z)=B(z) \overline{r(1 / \bar{z})}$, we get from (33) that, for $|z| \leq 1$,

$$
\overline{\mid r\left(\frac{1}{\bar{z}}\right)} \mid \leq \frac{\|r\|}{|B(z)|} \cdot \frac{\|r\||z|+\left|r^{*}(0)\right|}{\left|r^{*}(0)\|z \mid+\| r \|\right.},
$$

which clearly gives that, for $|z| \geq 1$,

$$
|r(z)| \leq \frac{\|r\|}{|B(1 / \bar{z})|} \cdot \frac{\|r\|+\left|r^{*}(0) \| z\right|}{\left|r^{*}(0)\right|+\|r\||z|} .
$$

It is clear from the definition of $B(z)$ that $|B(1 / \bar{z})|=$ $1 /|B(z)|$ and this, when combined with (35), gives that, for $|z| \geq 1$,

$$
\begin{aligned}
|r(z)| & \leq\|r\||B(z)| \frac{\|r\|+\left|r^{*}(0) \| z\right|}{\left|r^{*}(0)\right|+\|r\||z|} \\
& =\|r\||B(z)|\left(1-\frac{\left(\|r\|-\left|r^{*}(0)\right|\right)(|z|-1)}{\left|r^{*}(0)\right|+\|r\||z|}\right),
\end{aligned}
$$

which is (12) and the proof of Theorem 3 is thus complete.

\section{Data Availability}

No data were used to support this study.

\section{Conflicts of Interest}

The authors declare that they have no conflicts of interest.

\section{References}

[1] M. Abbas, A. A. Majid, and J. M. Ali, "Monotonicity-preserving $\mathrm{C}^{2}$ rational cubic spline for monotone data," Applied Mathematics and Computation, vol. 219, no. 6, pp. 2885-2895, 2012.

[2] M. Abbas, A. A. Majid, and J. M. Ali, "Positivity-preserving rational bi-cubic spline interpolation for $3 \mathrm{D}$ positive data," Applied Mathematics and Computation, vol. 234, pp. 460-476, 2014.

[3] N. Ankeny and T. Rivlin, "On a theorem of S. Bernstein," Pacific Journal of Mathematics, vol. 5, no. 6, pp. 849-852, 1955.

[4] N. K. Govil and R. N. Mohapatra, "Inequalities of maximum modulus of rational functions with prescribed poles," in Approximation Theory: In the Memory of A. K. Varma, N. K. Govil, R. N. Mohapatra, Z. Nashed, A. Sharma, and J. Szabados, Eds., Marcel Dekker, New York, NY, USA, 1998.

[5] N. K. Govil, "On the maximum modulus of polynomials not vanishing inside the unit circle," Approximation Theory and its Applications, vol. 5, pp. 79-82, 1989.

[6] A. Mir, "Some inequalities for maximum modulus of rational functions," Annales Universitatis Mariae Curie-Sklodowska, sectio A-Mathematica, vol. 73, no. 1, pp. 33-39, 2019.

[7] A. Aziz and Q. M. Dawood, "Inequalities for polynomials and its derivatives," Journal of Approximation Theory, vol. 54, pp. 306-313, 1998.

[8] G. V. Milovanović and A. Mir, "Estimates for the maximal modulus of rational functions with prescribed poles," Mathematical Notes, to Appear.

[9] Z. Nehari, Conformal Mapping, McGraw-Hill, New York, NY, USA, 1st edition, 1952.

[10] N. K. Govil, Q. I. Rahman, and G. Schmeisser, "On the derivative of a polynomial," Illinois Journal of Mathematics, vol. 23, pp. 319-329, 1979.

[11] R. Osserman, "A sharp Schwarz inequality on the boundary," Proceedings of the American Mathematical Society, vol. 128, no. 12, pp. 3513-3517, 2000. 